\title{
Anatase-silica composite aerogels: a nanoparticle-based approach
}

\section{Journal Article}

\section{Author(s):}

Heiligtag, Florian J.; Kränzlin, Niklaus; Süess, Martin J.; Niederberger, Markus (i)

Publication date:

2014-05

Permanent link:

https://doi.org/10.3929/ethz-b-000077154

\section{Rights / license:}

In Copyright - Non-Commercial Use Permitted

\section{Originally published in:}

Journal of Sol-Gel Science and Technology 70(2), https://doi.org/10.1007/s10971-013-3232-1 


\title{
Anatase-silica composite aerogels: a nanoparticle-based approach
}

\author{
Florian J. Heiligtag • Niklaus Kränzlin • \\ Martin J. Süess • Markus Niederberger
}

Received: 30 July 2013/Accepted: 2 December 2013/Published online: 11 December 2013

(C) Springer Science+Business Media New York 2013

\begin{abstract}
Herein we present the synthesis of anatasesilica aerogels based on the controlled gelation of preformed nanoparticle mixtures. The monolithic aerogels with macroscopic dimensions show large specific surface areas, and high and uniform porosities. The major advantage of such a particle-based approach is the great flexibility in pre-defining the compositional and structural features of the final aerogels before the gelation process by fine-tuning the properties of the titania and silica building blocks (e.g., size, composition and crystallinity) and their relative ratio in the dispersion. Specific surface functionalization enables control over the interaction between the nanoparticles and thus over their distribution in the aerogel. Positively charged titania nanoparticles are co-assembled with negatively charged Stoeber particles, resulting in a binary aerogel with a crystalline anatase and amorphous silica framework directly after supercritical drying without any calcination step. Titania-silica aerogels combine the photocatalytic activity of the anatase nanoparticles with the extensive silica chemistry available for silica surface functionalization.
\end{abstract}

Keywords Nanoparticles · Sol-gel chemistry · Aerogels $\cdot$ Silica $\cdot$ Titania $\cdot$ Self-assembly

F. J. Heiligtag · N. Kränzlin · M. J. Süess · M. Niederberger ( $₫)$ Laboratory for Multifunctional Materials, Department of Materials, ETH Zurich, Wolfgang-Pauli-Str. 10, 8093 Zurich, Switzerland

e-mail: markus.niederberger@mat.ethz.ch

M. J. Süess

Electron Microscopy ETH Zurich (EMEZ), Wolfgang-Pauli-Str. 16, 8093 Zurich, Switzerland

\section{Introduction}

Intelligent combination of well-known materials, their functionalities and their properties is, apart from the synthesis of entirely new materials, the most important design strategy for improved materials in materials chemistry. In recent years, especially the tuning of photocatalytically active materials like $\mathrm{TiO}_{2}$ attracted attention because of the enormous significance of these materials in the fields of energy conversion and chemical synthesis. There are numerous ways of improving the performance of catalysts, for example via modifying the composition by doping [1-4] or by using cocatalysts [1, 5-7]. But also optimization of the morphology (surface area and porosity) [8-10], the particle size [1, 11, 12], and the crystallinity $[13,14]$ offers various possibilities. Interesting results have been achieved, e.g., for mesoporous silica-titania composite materials [15-17]. They take advantage of both the beneficial morphology of the mesoporous silica framework with its high porosity and large surface area and the excellent photocatalytical properties of $\mathrm{TiO}_{2}$. Furthermore, the high photocatalytic activity of the $\mathrm{Si}-$ $\mathrm{O}-\mathrm{Ti}$ bond offers a synergetic effect, which gives additional reasons to combine titania and silica in one material $[18,19]$. However, the widespread use of these multicomponent photocatalysts is strongly limited by their accessibility via sol-gel routes. Simultaneous hydrolysis and condensation of two different precursors into a homogeneous gel is rather difficult. In the case of titaniasilica composites, titania alkoxides are typically more reactive than the silica precursors. The amount of titania, which can be incorporated in the final product is restricted, because silica typically forms the mesoporous structural backbone of the material. Furthermore, the rich surface chemistry of silica particles [20-22] cannot fully be exploited in a one-pot approach without considerably affecting the sol-gel processes. 
A particle-based approach with nanoparticles as building blocks could in principle overcome many of these limitations. Nanoparticles with a complex design, including doped materials, core-shell structures or particles with specific surface functionality can first be prepared and then, in a subsequent step, be assembled into a 3-dimensionally connected network. It was already shown that a controlled destabilization of nanoparticle dispersions can result in gels and aerogels [23, 24]. Aqueous dispersions of 2-amino-2(hydroxymethyl)-1,3-propanediol (trizma) functionalized anatase nanoparticles were destabilized in a controlled way by the desorption of trizma from the $\{001\}$ facets of anatase $[25,26]$. The result of this process is that the anatase nanoparticles undergo oriented attachment along the [001] direction. If the particle concentration in the dispersion is high enough, these pearl-necklace-like arrangements branch out and fill the whole reaction vessel, creating a continuous and macroscopic gel [27].

Here we present the preparation of a nanoparticle based anatase-silica aerogel as a model system of a binary material with complementary properties. The approach takes advantage of the gelation behavior of surface-functionalized anatase nanoparticles, which can be used as host matrix for embedding the silica nanoparticles, giving access to aerogels with high titania content. Adjustment of the zeta potential of both building blocks ensures a homogeneous distribution of the silica particles in the gel, a fast gelation process and a good adhesion of both building blocks to each other. After supercritical drying, monolithic aerogels with a crystalline titania backbone and embedded amorphous silica particles are obtained.

\section{Experimental details}

\subsection{Chemicals}

Titanium(IV) chloride (99.9\%), tetraethyl orthosilicate (TEOS, $98 \%$ ), aqueous ammonia solution ( $25 \%$ ), benzyl alcohol (puriss., 99-100.5\% GC), chloroform ( $\geq 99.8 \%$ ), acetone $(>99.5 \%)$ and diethyl ether $(\geq 99.8 \%)$ were purchased from Sigma-Aldrich. 2-Amino-2-(hydroxymethyl)1,3-propanediol (trizma, $\geq 99.7 \%$ ) was purchased from Fluka. Ethanol (analytical grade) was purchased from Scharlau, Spain. Liquid carbon dioxide was provided by PanGas AG, Switzerland. All chemicals were used without further purification.

\subsection{Synthesis}

Synthesis of trizma functionalized anatase nanoparticles: the synthesis of trizma functionalized anatase nanoparticles was performed according to previously published protocols
[25, 26]. In brief, $3.42 \mathrm{mmol}$ (414 mg) 2-amino-2(hydroxymethyl)-1,3-propanediol (trizma) was dissolved in $90 \mathrm{ml}$ benzyl alcohol under continuous stirring by heating to $80{ }^{\circ} \mathrm{C}$ for $15 \mathrm{~min}$. After cooling to room temperature, $41.0 \mathrm{mmol}(4.5 \mathrm{ml}) \mathrm{TiCl}_{4}$ was added dropwise under vigorous stirring. The reaction mixture was heated to $80^{\circ} \mathrm{C}$ for $24 \mathrm{~h}$ and cooled to room temperature. The colorless, precipitated product was separated from the yellowish reaction mixture by centrifugation and washed by redispersing and centrifugation five times with chloroform and twice with diethyl ether. The colorless product was finally redispersed in $30 \mathrm{ml} \mathrm{H}_{2} \mathrm{O}$ and residues of diethyl ether were removed by applying vacuum.

Synthesis of silica nanoparticles: Silica nanoparticles were synthesized by the Stöber process [30] according to a previously published protocol [21]. $50 \mathrm{ml}$ ethanol, $1.0 \mathrm{ml}$ $\mathrm{H}_{2} \mathrm{O}$ and $1.0 \mathrm{ml}$ aqueous ammonia solution $(25 \%)$ were mixed and heated to $40{ }^{\circ} \mathrm{C}$. $6.73 \mathrm{mmol}(1.5 \mathrm{ml})$ TEOS was slowly added to the solvent mixture and continuously stirred for $3 \mathrm{~h}$. Subsequently, additional $4.48 \mathrm{mmol}$ $(1.0 \mathrm{ml})$ TEOS were dropped to the solution and stirred for $3 \mathrm{~h}$ at $40{ }^{\circ} \mathrm{C}$. The reaction solution was cooled to room temperature and aged for 1 week.

Gelation and aerogel preparation: $10 \mathrm{ml}$ of an aqueous dispersion of trizma functionalized anatase nanoparticles and $10 \mathrm{ml}$ of an ethanolic silica nanoparticle dispersion were mixed and poured in a Teflon casting mold. The mold was sealed and heated to $60{ }^{\circ} \mathrm{C}$ for $30 \mathrm{~min}$ to induce the gelation process. The molds were cooled to room temperature and the monolithic gels were removed. A solvent exchange process was carried out from a water-ethanol mixture to acetone in steps of $10 \mathrm{vol} \%$ per day. Supercritical drying was performed with supercritical $\mathrm{CO}_{2}$ using a Leica CPD 030.

\subsection{Characterization}

Powder X-ray diffraction was performed on a Panalytical Empyrean diffractometer using $\mathrm{Cu} \mathrm{K}$-alpha radiation. For measuring the aerogel sample, pieces of the monolithic sample were ground in an agate mortar to obtain a powder. Scanning electron microscopy was carried out on a FEI Magellan 400 in immersion mode using $2.00 \mathrm{kV}$ and 0.20 $\mathrm{nA}$. To avoid any changes of the fragile and fine aerogel structure, the samples were used without sputtering. For transmission electron microscopy (TEM) in Fig. 4a, energy dispersive X-ray spectroscopy (EDX) and selected area electron diffraction (SAED) a Philips Tecnai F30 operated at $300 \mathrm{kV}$ was used. For TEM in Fig. 4b, c, a Philips CM 200 operated at $160 \mathrm{kV}$ was used. For sample preparation some drops of chloroform were added to a small amount of aerogel, mixed with a spatula and dropped on a carbon coated copper grid placed on a filter paper to remove 
excess liquid. Zeta potential was measured on a Malvern Zetasizer Nano-ZS. A diluted dispersion of the trizma functionalized anatase nanoparticles was made by adding 3 drops of dispersion in $10 \mathrm{ml}$ of water. The $\mathrm{pH}$ was adjusted by adding sodium hydroxide solution or hydrochloric acid. Attenuated total reflectance infrared (ATR-IR) spectroscopy was performed on a Bruker Alpha FT-IR Spectrometer with diamond ATR optics. For the measurement, the aerogel samples were ground in an agate mortar. Nitrogen sorption experiments were carried out on a Quantachrome Autosorb-iQ-C-XR at $77 \mathrm{~K}$, with nitrogen $(99.999 \%)$ and helium (99.999 \%) provided by PanGas AG, Switzerland. Before the measurement, the samples were outgassed in vacuum at $100{ }^{\circ} \mathrm{C}$ for at least $6 \mathrm{~h}$.

\section{Results and discussion}

The surface chemistry of the nanoparticle building blocks plays a major role during the gelation process. As previously reported, gelation of trizma functionalized anatase nanoparticles can be induced by desorption of trizma molecules from the $\{001\}$ facets of the anatase nanoparticles, which induces an oriented attachment process [27]. In dispersions with low concentrations of nanoparticles long pearl necklace like chains of anatase nanoparticles oriented in [001] direction can be observed [25, 26]. In higher concentrated nanoparticle dispersions a branching of the linear assemblies leads to a 3-dimensional network, a gel [27]. In the case of a binary titania-silica nanoparticle mixture, a second mechanism comes into play during the gelation of such a co-dispersion: Electrostatic attraction between silica nanoparticles and the trizma functionalized anatase nanoparticles. This becomes obvious when having a look at the isoelectric points (IEP) of the components. Silica has an IEP around $\mathrm{pH} 2$ [28]. The IEP of anatase is reported to be around 5.8 [29]. Measurements of the zeta potential show an additional effect of the trizma functionalization on the surface charge of the anatase nanoparticles. The IEP of the particles is shifted to higher $\mathrm{pH}$ values of 7-8, enhancing the electrostatic attraction of the two types of nanoparticle building blocks. The simultaneous occurrence of oriented attachment and electrostatic attraction results in faster gelation times even at low temperatures, a noticeable increase in viscosity already during mixing of both nanoparticle dispersions and good adhesion between the two building blocks.

The powder X-ray diffraction (XRD) patterns of a titania-silica composite aerogel and the titania and silica building blocks are shown in Fig. 1. In spite of the broad reflections due to the small crystal size, the diffraction peaks can clearly be assigned to anatase phase (ICDD File card No. 1-70-6826). The diffractogram of the silica

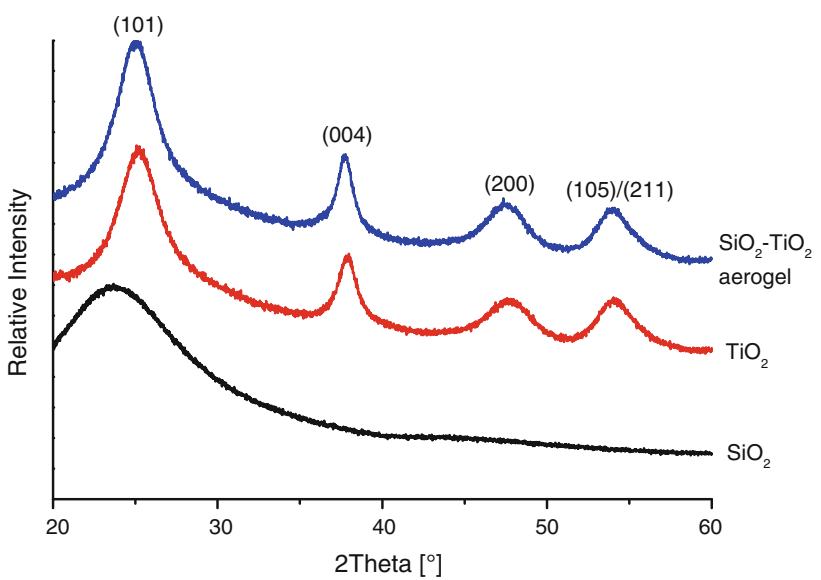

Fig. 1 XRD patterns of the silica nanoparticle powder, the trizma functionalized anatase nanoparticle powder and the silica-anatase aerogel

particles shows, as expected for an amorphous material, only one broad hump. Silica nanoparticles made by the Stoeber method [30] are in general amorphous. Consequently, the diffractogram of the composite aerogel exhibits only the reflections of the anatase building blocks. Using Scherrer's equation for the calculation of the crystallite size in [101] direction gives a value of $2.9 \mathrm{~nm}$.

Attenuated total reflection infrared (ATR-IR) spectroscopy was performed on dried powders of as-synthesized silica and anatase nanoparticles and on the final anatasesilica composite aerogel (Fig. 2). All spectra show rather small features in the range from $4,000-1,700 \mathrm{~cm}^{-1}$, which originate from traces of water/OH and alkyl and/or aryl species present [31]. The spectrum of the silica nanoparticles shows a weak band at $1,630 \mathrm{~cm}^{-1}$, which corresponds to stretching vibrations of silica [32]. In the region from 1,200-400 $\mathrm{cm}^{-1}$ much stronger peaks were detected. The strong band at $1,065 \mathrm{~cm}^{-1}$ and its shoulder at $1,200 \mathrm{~cm}^{-1}$, the peaks at $800 \mathrm{~cm}^{-1}$, at 450 and at $555 \mathrm{~cm}^{-1}$ originate from stretching vibrations, bending vibrations and rocking vibrations of $\mathrm{Si}-\mathrm{O}-\mathrm{Si}$ groups, respectively [33, 34]. The peak at $955 \mathrm{~cm}^{-1}$ can be assigned to $\mathrm{Si}-\mathrm{OH}$ stretching vibrations, the small peak at $875 \mathrm{~cm}^{-1}$ to a Si-OH bending vibration [35].

The infrared spectrum of trizma functionalized anatase nanoparticles shows in the area of $1,700-400 \mathrm{~cm}^{-1}$ signals from the titanium dioxide lattice, adsorbed water and traces of benzyl alcohol and trizma from the synthesis. Sharp peaks at 1,492 and 1,452 $\mathrm{cm}^{-1}$ arise from $\mathrm{C}-\mathrm{H}$ deformation vibrations probably from the aromatic ring of benzyl alcohol. Peaks at 1,065 and $1,105 \mathrm{~cm}^{-1}$ can be assigned to $\mathrm{C}-\mathrm{O}$ stretching vibrations from the $\mathrm{OH}$ groups of trizma and benzyl alcohol. The broad peak around $1,610 \mathrm{~cm}^{-1}$ originates from $\mathrm{H}-\mathrm{O}-\mathrm{H}$ bending vibrations of weakly bound water adsorbed on the titania powder [35, 36]. Ti- 

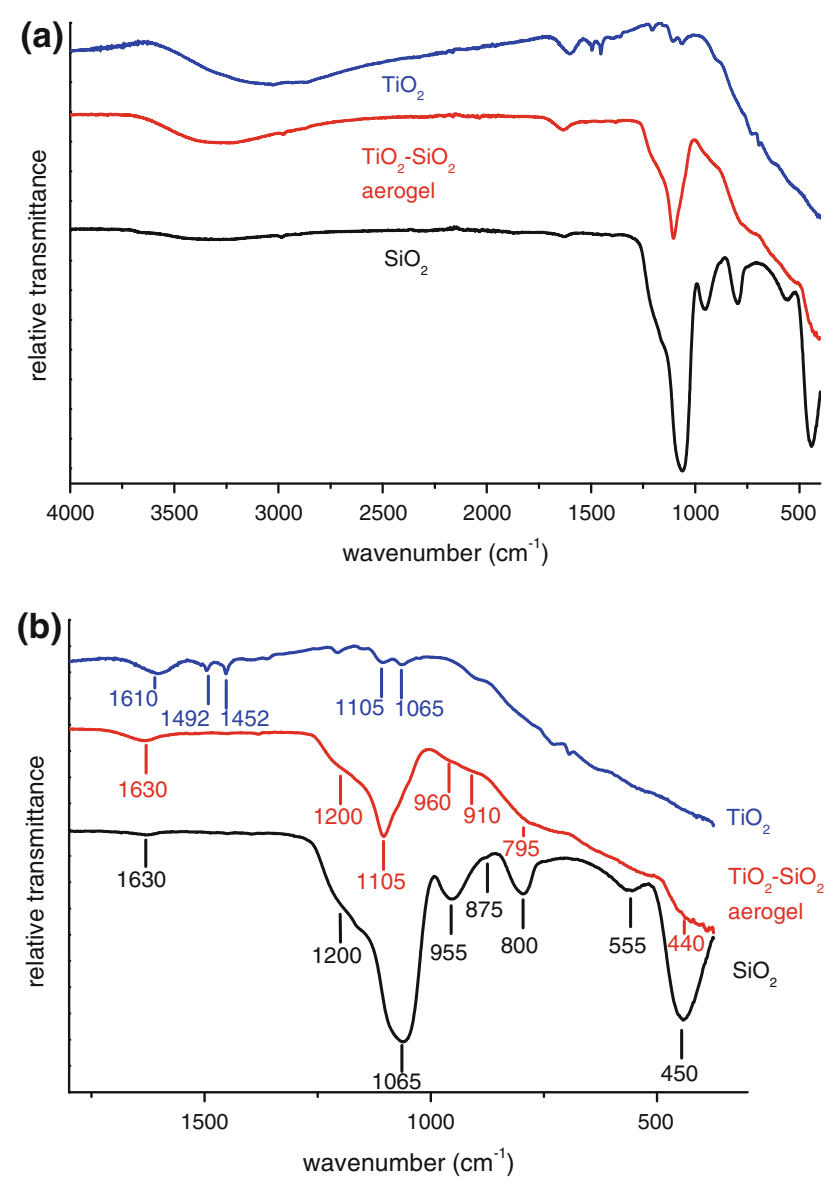

Fig. 2 Infrared spectra of silica nanoparticles, trizma functionalized anatase nanoparticles and a titania-silica aerogel. a Overview spectrum and $\mathbf{b}$ magnified range at lower wavenumbers

$\mathrm{O}-\mathrm{Ti}$ vibrations and $\mathrm{Ti}-\mathrm{OH}$ vibrations are expected at 653-550 and 495-436 $\mathrm{cm}^{-1}$, respectively [37]. In this range the IR spectrum shows a very strong signal with several shoulders. The limited resolution of the vibrations does not allow to unambiguously assign every single peak, but the intensity of this broad band gives clear evidence for the presence of $\mathrm{Ti}-\mathrm{O}-\mathrm{Ti}$ and $\mathrm{Ti}-\mathrm{OH}$ groups.

The IR spectrum of the composite aerogel shows a combination of features of both IR spectra of silica and titania. A small, broad peak at $1,630 \mathrm{~cm}^{-1}$ corresponds to $\mathrm{H}-\mathrm{O}-\mathrm{H}$ deformation vibrations from weakly bound water on the aerogel surface. Signals from silica can be found in the range from 1,250 to $370 \mathrm{~cm}^{-1}$. The peak at $1,105 \mathrm{~cm}^{-1}$ and its shoulder at $1,200 \mathrm{~cm}^{-1}$ stem from $\mathrm{Si}-\mathrm{O}-\mathrm{Si}$ stretching vibrations. The $\mathrm{Si}-\mathrm{O}-\mathrm{Si}$ bending and rocking vibrations at 795 and $440 \mathrm{~cm}^{-1}$ can only be observed as shoulders of a strong peak, which ranges from $995 \mathrm{~cm}^{-1}$ to the end of the measurement range. This is the characteristic peak of the Ti-O-Ti and Ti-OH vibrations. Accordingly, the IR spectrum proves the presence of silica and titania in the final composite aerogel. The typical vibrations of $\mathrm{Ti}-$
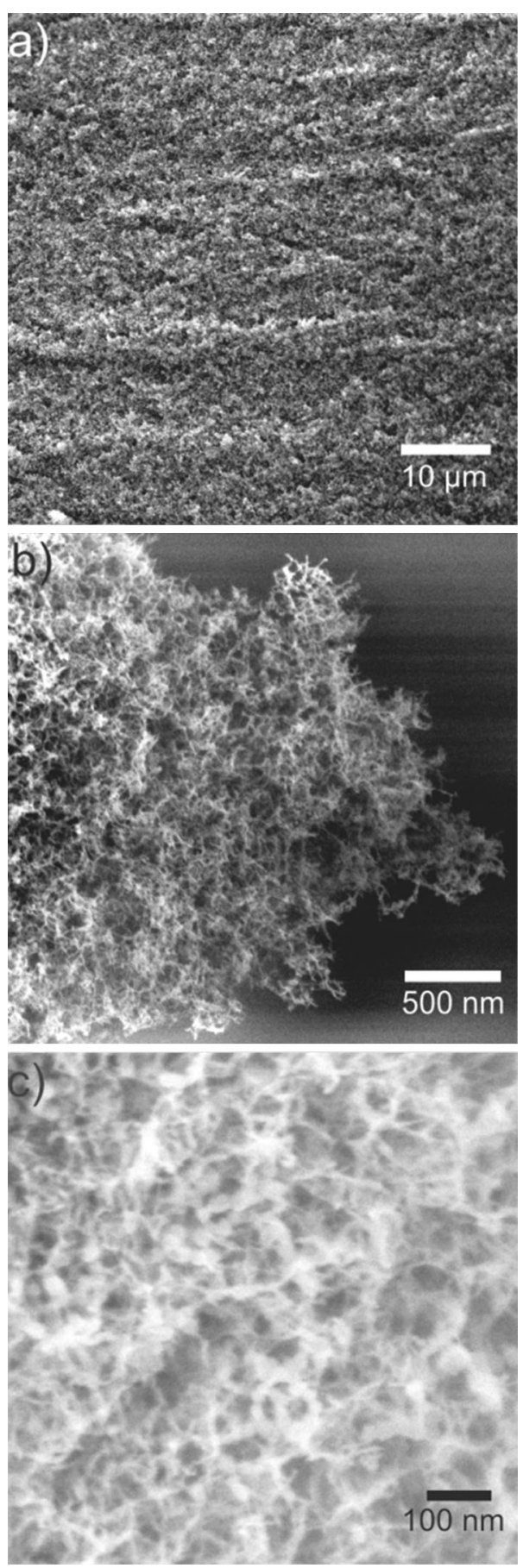

Fig. 3 SEM images of a silica-titania aerogel $\mathbf{a}$ at low and $\mathbf{b}, \mathbf{c}$ at higher magnifications

O-Si at $910 \mathrm{~cm}^{-1}$ cannot clearly be distinguished [38, 39]. The small shoulders at 960 and $910 \mathrm{~cm}^{-1}$ could be assigned to $\mathrm{Si}-\mathrm{OH}$ and $\mathrm{Ti}-\mathrm{O}-\mathrm{Si}$ vibrations, respectively. In any case, the peak intensity of the $910 \mathrm{~cm}^{-1}$ peak is not expected to be very intense, because in the present case there is no homogeneous silica-titania glass, but a mixture of discrete $\mathrm{SiO}_{2}$ and $\mathrm{TiO}_{2}$ nanoparticles with less close contact between the building blocks. 

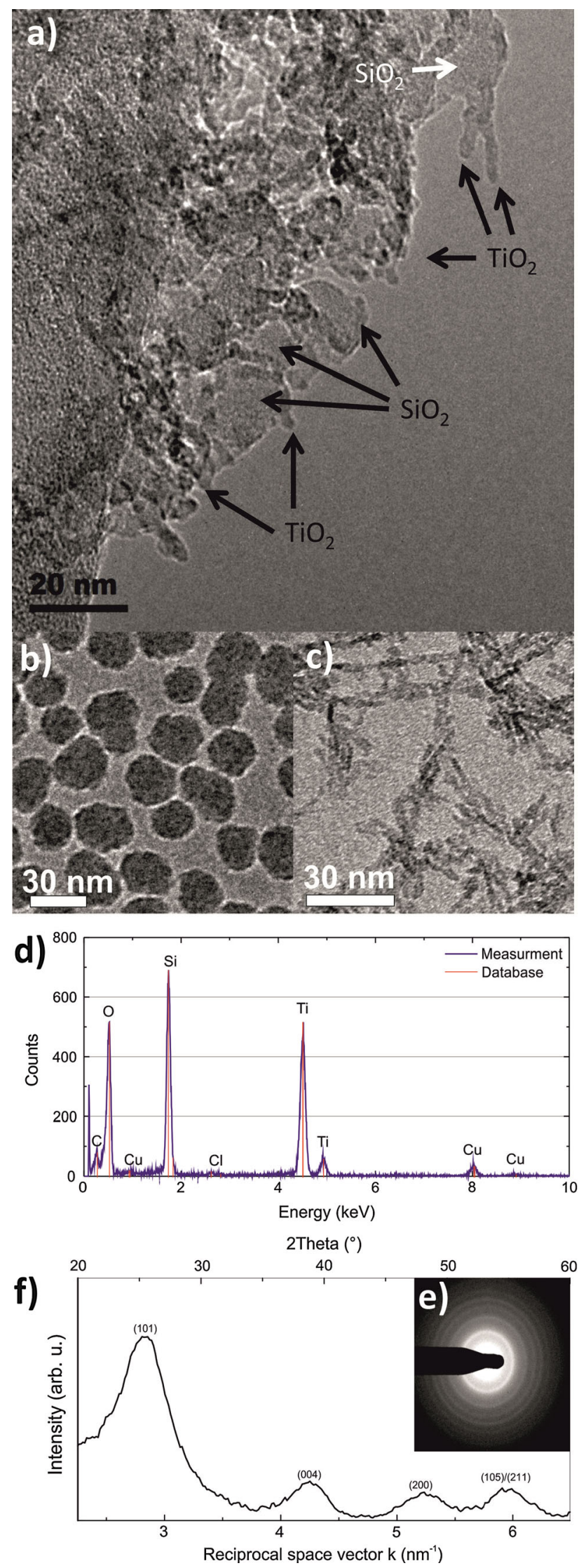

Fig. 4 Transmission electron micrographs of a $\mathrm{SiO}_{2}-\mathrm{TiO}_{2}$ aerogel, $\mathbf{b}$ as synthesized silica nanoparticles and $\mathbf{c}$ linear oriented attachments of trizma functionalized anatase nanoparticles. d EDX spectrum of the sample. e SAED of the sample and $\mathbf{f}$ line profile of the SAED fitted to anatase

An overview scanning electron microscopy (SEM) image of the anatase-silica composite aerogel shows the uniform pore structure of the sample extending homogeneously over several tens of microns (Fig. 3a). At higher magnification the 3-dimensional network becomes clearly visible (Fig. 3b). Fine threads of anatase nanoparticles build up the fluffy morphology. Further increase in magnification allows to distinguish the silica nanoparticles from the very fine pearl-necklace-like arrangements of the titania nanoparticles just a few nanometre thick (Fig. 3c). The titania nanoparticles are assembled into straight sections as well as into branched structures, incorporating the nearly spherical silica nanoparticles with diameters of about $20 \mathrm{~nm}$.

Transmission electron microscopy (TEM) investigations support the presence of two types of nanoparticles (Fig. 4a). The larger silica nanoparticles show a lower contrast in comparison to anatase nanoparticles. The concept of nanoparticle assembly by electrostatic attraction between silica and anatase nanoparticles as well as the oriented attachment between anatase nanoparticles is nicely illustrated in the TEM image. Due to electrostatic attraction between silica and titania, the silica nanoparticles are partly coated by the titania nanoparticles. At the same time, the titania nanoparticles form 1-dimensional structure segments typical for oriented attachment (particularly nicely visible in the upper right corner of Fig. 4a). For better distinction of silica and titania nanoparticles in the TEM, images of oriented attached, trizma functionalized anatase nanoparticles and as synthesized silica nanoparticles are shown in Fig. $4 \mathrm{~b}$ and c, respectively. The linear aggregates of small $3 \mathrm{~nm}$ anatase particles can clearly be distinguished from the $20-30 \mathrm{~nm}$ spherical silica particles. Energy dispersive X-ray spectroscopy (EDX) (Fig. 4d) shows the presence of silicon, titanium and oxygen. The chloride impurities are a result of the titanium tetrachloride used as precursor. The copper signal is due to the TEM grid. Selected area electron diffraction (SAED) (Fig. 4e) shows a crystalline system with amorphous contributions. The amorphous contributions are supposed to come from the silica. Analyzing a line projection of the diffraction pattern (Fig. 4f) the contributions from the crystalline building block match the diffractogram of anatase.

Nitrogen sorption experiments were performed on the supercritically dried anatase-silica aerogel (Fig. 5a). The BET specific surface area is $488 \mathrm{~m}^{2} / \mathrm{g}$, which seems to be a typical result for a silica-titania aerogel [17]. But 

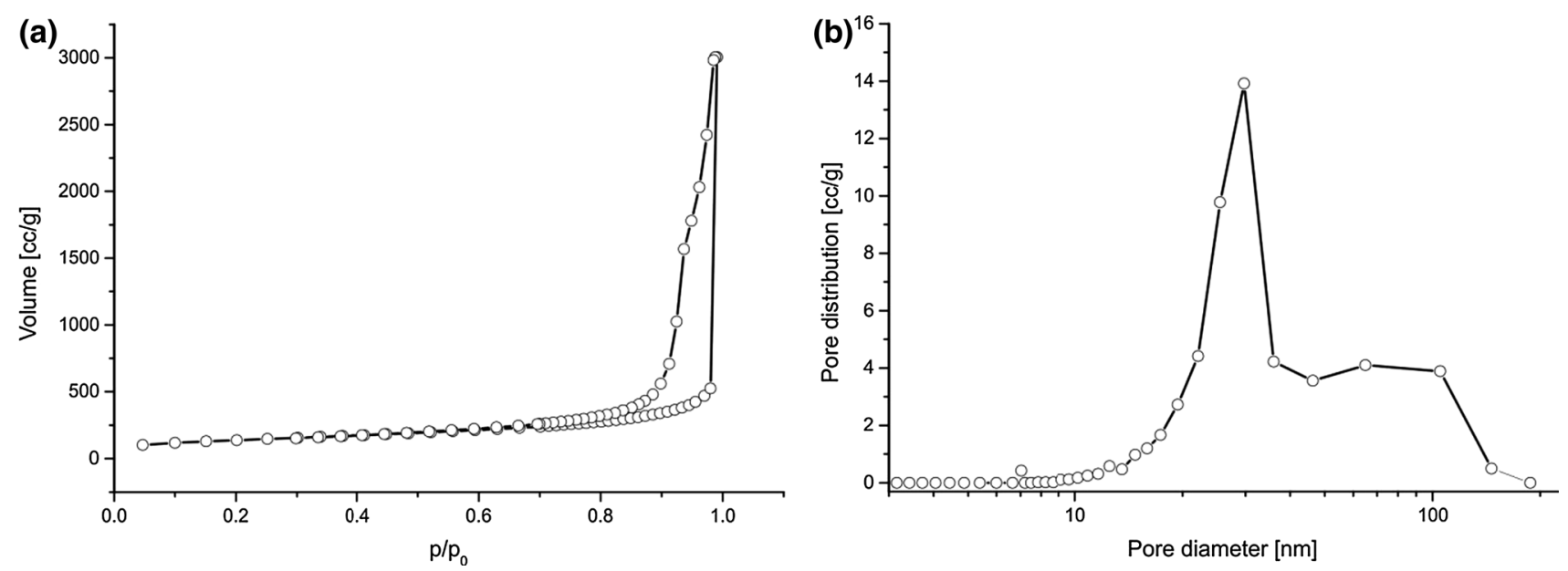

Fig. 5 a Nitrogen adsorption-desorption isotherm of a $\mathrm{TiO}_{2}-\mathrm{SiO}_{2}$ aerogel. b Pore size distribution $\mathrm{dV}(\log (\mathrm{d}))$ calculated from the nitrogen desorption data according to the BJH-method

considering that titania is the major component in our aerogel, such a surface area is rather high. An aerogel with a Ti-to-Si ratio of 1 was reported to exhibit a surface area of $230 \mathrm{~m}^{2} / \mathrm{g}$ [40]. The BJH analysis of the desorption branch of the $\mathrm{N}_{2}$ sorption experiment showed an average pore diameter of $38 \mathrm{~nm}$. The mode of the pore diameter is $30 \mathrm{~nm}$ (Fig. 5b). The pore diameter is comparable with titania-silica aerogels synthesized by classical sol-gel approach and supercritically dried with $\mathrm{CO}_{2}$ [41]. However, the pore diameters of titania and titania-silica aerogels can vary in a wide spectrum [42]. The total pore volume is $4.66 \mathrm{~cm}^{3} / \mathrm{g}$ for pores with a diameter smaller than $216 \mathrm{~nm}$. The lack of a clear plateau region especially in the adsorption branch suggests a higher total pore volume outside the measurement range.

\section{Conclusion}

Here we showed an easy way to prepare an anatase-silica composite aerogel by co-gelation of preformed nanoparticles. This approach gives access to aerogels with high titania contents. Additionally, the use of nanoparticles as building blocks offers the unique advantage to obtain a crystalline porous network without the need of a calcination step. Accordingly, the problem of pore shrinkage and monolith cracking often observed during an annealing step is avoided. The co-assembly of titania with silica represents a proof-of-concept, offering two important features that are hard to achieve by classical sol-gel chemistry: (1) simultaneous incorporation of amorphous and crystalline nanoparticles, and (2) combination of two different properties, namely photocatalytic activity from the anatase nanocrystals and great variability of surface chemistry from the silica particles. Nanoparticle-based approaches provide a flexible and modular access to aerogels with pre-defined compositional and structural features, greatly extending the functionalities of these fascinating materials.

Acknowledgments Financial support by ETH Zürich (ETH-07 09-2) is gratefully acknowledged. We thank the Electron Microscopy Center EMEZ of ETH Zürich for access to TEM and SEM facilities, as well as the Laboratory of Surface Science and Technology, Department of Materials, ETH Zurich for access to the Malvern Zetasizer Nano-ZS. We further thank Linda Oberli for her work in the lab and Philippe Knüsel for the measurement of the Zeta potentials.

\section{References}

1. Zhang ZB, Wang CC, Zakaria R, Ying JY (1998) Role of particle size in nanocrystalline $\mathrm{TiO}_{2}$-based photocatalysts. J Phys Chem B 102(52):10871-10878. doi:10.1021/jp982948

2. Asahi R, Morikawa T, Ohwaki T, Aoki K, Taga Y (2001) Visible-light photocatalysis in nitrogen-doped titanium oxides. Science 293(5528):269-271. doi:10.1126/science.1061051

3. Choi WY, Termin A, Hoffmann MR (1994) The role of metal-ion dopants in quantum-sized $\mathrm{TiO}_{2}$ - Correlation between photoreactivity and charge-carrier recombination dynamics. J Phys Chem 98(51):13669-13679. doi:10.1021/j100102a038

4. Djerdj I, Arcon D, Jaglicic Z, Niederberger M (2008) Nonaqueous synthesis of metal oxide nanoparticles: Short review and doped titanium dioxide as case study for the preparation of transition metal-doped oxide nanoparticles. J Solid State Chem 181(7):1571-1581. doi:10.1016/j.jssc.2008.04.016

5. Zhao W, Chen CC, Li XZ, Zhao JC, Hidaka H, Serpone N (2002) Photodegradation of sulforhodamine-B dye in platinized titania dispersions under visible light irradiation: influence of platinum as a functional co-catalyst. J Phys Chem B 106(19):5022-5028. doi:10.1021/jp020205p

6. Yu JG, Ran JR (2011) Facile preparation and enhanced photocatalytic $\mathrm{H}_{2}$-production activity of $\mathrm{Cu}(\mathrm{OH})_{2}$ cluster modified $\mathrm{TiO}_{2}$. Energy Environ Sci 4(4):1364-1371. doi:10.1039/c0ee00729c

7. Kato S, Hirano Y, Iwata M, Sano T, Takeuchi K, Matsuzawa S (2005) Photocatalytic degradation of gaseous sulfur compounds by silver-deposited titanium dioxide. Appl Catal B 57(2): 109-115. doi:10.1016/j.apcatb.2004.10.015 
8. Pajonk GM (1997) Catalytic aerogels. Catal Today 35(3): 319-337. doi:10.1016/s0920-5861(96)00163-0

9. Rolison D (2003) Catalytic nanoarchitectures-The importance of nothing and the unimportance of periodicity. Science 299(5613): 1698-1701

10. Schneider M, Baiker A (1995) Aerogels in catalysis. Catal Rev Sci Eng 37(4):515-556. doi:10.1080/01614949508006450

11. Maira AJ, Yeung KL, Lee CY, Yue PL, Chan CK (2000) Size effects in gas-phase photo-oxidation of trichloroethylene using nanometer-sized $\mathrm{TiO}_{2}$ catalysts. J Catal 192(1):185-196. doi:10. 1006/jcat 2000.2838

12. Maira AJ, Yeung KL, Soria J, Coronado JM, Belver C, Lee CY (2001) Gas-phase photo-oxidation of toluene using nanometersize $\mathrm{TiO}_{2}$ catalysts. Appl Catal B 29(4):327-336. doi:10.1016/ s0926-3373(00)00211-3

13. Gao L, Zhang QH (2001) Effects of amorphous contents and particle size on the photocatalytic properties of $\mathrm{TiO}_{2}$ nanoparticles. Scr Mater 44(8-9):1195-1198. doi:10.1016/s1359-6462(01) 00681-9

14. Leung DYC, Fu XL, Wang CF, Ni M, Leung MKH, Wang XX, Fu XZ (2010) Hydrogen production over titania-based photocatalysts. ChemSusChem 3(6):681-694. doi:10.1002/cssc.201000014

15. Yao N, Cao SL, Yeung KL (2009) Mesoporous $\mathrm{TiO}_{2}-\mathrm{SiO}_{2}$ aerogels with hierarchal pore structures. Microporous Mesoporous Mater 117(3):570-579. doi:10.1016/j.micromeso.2008.08.020

16. Kibombo HS, Peng R, Rasalingam S, Koodali RT (2012) Versatility of heterogeneous photocatalysis: Synthetic methodologies epitomizing the role of silica support in $\mathrm{TiO}_{2}$ based mixed oxides. Catal Sci Tech 2(9):1737-1766. doi:10.1039/c2cy20247f

17. Ruzimuradov O, Nurmanov S, Kodani Y, Takahashi R, Yamada I (2012) Morphology and dispersion control of titania-silica monolith with macro-meso pore system. J Sol-Gel Sci Technol 64(3):684-693. doi:10.1007/s10971-012-2903-7

18. Gao XT, Wachs IE (1999) Titania-silica as catalysts: molecular structural characteristics and physico-chemical properties. Catal Today 51(2):233-254. doi:10.1016/s0920-5861(99)00048-6

19. Anderson C, Bard AJ (1997) Improved photocatalytic activity and characterization of mixed $\mathrm{TiO}_{2} / \mathrm{SiO}_{2}$ and $\mathrm{TiO}_{2} / \mathrm{Al}_{2} \mathrm{O}_{3}$ materials. J Phys Chem B 101(14):2611-2616. doi:10.1021/jp9626982

20. Qhobosheane M, Santra S, Zhang P, Tan WH (2001) Biochemically functionalized silica nanoparticles. Analyst 126(8): 1274-1278. doi:10.1039/b101489g

21. Achatz DE, Heiligtag FJ, Li XH, Link M, Wolfbeis OS (2010) Colloidal silica nanoparticles for use in click chemistry-based conjugations and fluorescent affinity assays. Sens Actuators B 150(1):211-219. doi:10.1016/j.snb.2010.07.014

22. Wang L, Zhao W, Tan W (2008) Bioconjugated silica nanoparticles: development and applications. Nano Res 1(2):99-115. doi:10.1007/s12274-008-8018-3

23. Mohanan J, Arachchige I, Brock S (2005) Porous semiconductor chalcogenide aerogels. Science 307(5708):397-400. doi:10.1126/ science. 1104226

24. Gaponik N, Herrmann AK, Eychmuller A (2012) Colloidal nanocrystal-based gels and aerogels: material aspects and application perspectives. J Phys Chem Lett 3(1):8-17. doi:10.1021/ jz201357r

25. Polleux J, Pinna N, Antonietti M, Niederberger M (2004) Liganddirected assembly of preformed titania nanocrystals into highly anisotropic nanostructures. Adv Mater 16(5):436-439. doi:10. 1002/adma.200306251

26. Polleux J, Pinna N, Antonietti M, Hess C, Wild U, Schlögl R, Niederberger M (2005) Ligand functionality as a versatile tool to control the assembly behavior of preformed titania nanocrystals. Chem Eur J 11(12):3541-3551. doi:10.1002/chem.200401050

27. Heiligtag FJ, Rossell MD, Süess MJ, Niederberger M (2011) Template-free co-assembly of preformed $\mathrm{Au}$ and $\mathrm{TiO}_{2}$ nanoparticles into multicomponent 3D aerogels. J Mater Chem 21(42): 16893-16899. doi:10.1039/c1jm11740h

28. Iler RK (1979) The chemistry of silica. Wiley, New York

29. Kosmulski M (2003) A literature survey of the differences between the reported isoelectric points and their discussion. Colloids Surf Physicochem Eng Aspects 222(1-3):113-118. doi:10.1016/S0927-7757(03)00240-1

30. Stöber W, Fink A, Bohn E (1968) Controlled growth of monodisperse silica spheres in micron size range. J Colloid Interface Sci 26(1):62-69. doi:10.1016/0021-9797(68)90272-5

31. Kotsokechagia T, Cellesi F, Thomas A, Niederberger M, Tirelli N (2008) Preparation of ligand-free $\mathrm{TiO}_{2}$ (anatase) nanoparticles through a nonaqueous process and their surface functionalization. Langmuir 24(13):6988-6997. doi:10.1021/la800470e

32. Davis KM, Tomozawa M (1995) Water diffusion into silica glass-structural-changes in silica glass and their effect on water solubility and diffusivity. J Non-Cryst Solids 185(3):203-220. doi:10.1016/0022-3093(95)00015-1

33. Bell RJ, Bird NF, Dean P (1968) Vibrational spectra of vitreous silica germania and beryllium fluoride. J Phys C 1(2):299-303. doi: $10.1088 / 0022-3719 / 1 / 2 / 304$

34. Galeener FL, Lucovsky G (1976) Longitudinal optical vibrations in glasses- $\mathrm{GeO}_{2}$ and $\mathrm{SiO}_{2}$. Phys Rev Lett 37(22):1474-1478. doi:10.1103/PhysRevLett.37.1474

35. Davis KM, Tomozawa M (1996) An infrared spectroscopic study of water-related species in silica glasses. J Non-Cryst Solids 201(3):177-198. doi:10.1016/0022-3093(95)00631-1

36. Li GS, Li LP, Boerio-Goates J, Woodfield BF (2005) High purity anatase $\mathrm{TiO}_{2}$ nanocrystals: near room-temperature synthesis, grain growth kinetics, and surface hydration chemistry. J Am Chem Soc 127(24):8659-8666. doi:10.1021/ja050517g

37. Larbot A, Laaziz I, Marignan J, Quinson JF (1992) Porous texture of a titanium-oxide gel-evolution as a function of medium used. J Non-Cryst Solids 147:157-161. doi:10.1016/s0022-3093(05) 80610-6

38. Zeitler VA, Brown CA (1957) The infrared spectra of some TiO-Si, Ti-O-Ti and Si-O-Si compounds. J Phys Chem 61(9): 1174-1177. doi:10.1021/j150555a010

39. Aizawa M, Nosaka Y, Fujii N (1991) FT-IR liquid attenuated total reflection study of $\mathrm{TiO}_{2}-\mathrm{SiO}_{2}$ sol-gel reaction. J Non-Cryst Solids 128(1):77-85. doi:10.1016/0022-3093(91)90778-5

40. Rupp W, Hüsing N, Schubert U (2002) Preparation of silicatitania xerogels and aerogels by sol-gel processing of new single-source precursors. J Mater Chem 12(9):2594-2596. doi:10.1039/ b204956b

41. Dutoit DCM, Schneider M, Baiker A (1995) Titania-silica mixed oxides.1. Influence of sol-gel and drying conditions on structuralproperties. J Catal 153(1):165-176. doi:10.1006/jcat.1995.1118

42. Schneider M, Baiker A (1997) Titania-based aerogels. Catal Today 35(3):339-365. doi:10.1016/s0920-5861(96)00164-2 